\title{
FoxO mediates APP-induced AICD-dependent cell death
}

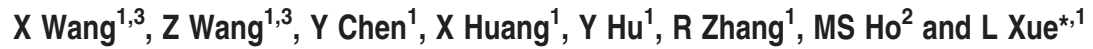

The amyloid precursor protein (APP) is a broadly expressed transmembrane protein that has a significant role in the pathogenesis of Alzheimer's disease (AD). APP can be cleaved at multiple sites to generate a series of fragments including the amyloid $\beta(A \beta)$ peptides and APP intracellular domain (AICD). Although A $\beta$ peptides have been proposed to be the main cause of $A D$ pathogenesis, the role of AICD has been underappreciated. Here we report that APP induces AICD-dependent cell death in Drosophila neuronal and non-neuronal tissues. Our genetic screen identified the transcription factor forkhead box 0 (Fox 0 ) as a crucial downstream mediator of APP-induced cell death and locomotion defect. In mammalian cells, AICD physically interacts with FoxO in the cytoplasm, translocates with FoxO into the nucleus upon oxidative stress, and promotes FoxO-induced transcription of pro-apoptotic gene Bim. These data demonstrate that APP modulates FoxO-mediated cell death through AICD, which acts as a transcriptional co-activator of FoxO.

Cell Death and Disease (2014) 5, e1233; doi:10.1038/cddis.2014.196; published online 15 May 2014

Subject Category: Neuroscience

Alzheimer's disease (AD), characterized by a progressive cognitive decline leading to social and occupational debilitation, has been regarded as the most prevalent neurodegenerative disease in the world. ${ }^{1} \mathrm{AD}$ is pathologically differentiated from other causes of dementia by a reduction in synaptic contact, ${ }^{2}$ accumulation of neurofibrillary tangles, ${ }^{3,4}$ aggregates of the hyper-phosphorylated microtubule-associated protein tau, ${ }^{5}$ and extracellular senile plaques (SPS) containing the amyloid $\beta$ peptide $(\mathrm{A} \beta) .{ }^{6}$ Although senior individuals often develop some plaques and tangles as a consequence of aging, the brains of $A D$ patients have a greater number of them in specific brain regions such as the temporal lobe. ${ }^{3}$

The $\mathrm{A} \beta$ peptide found predominantly in the SPs is generated from a large single-pass transmembrane protein termed as amyloid precursor protein (APP). ${ }^{7}$ Intriguingly, mutations in APP have been implicated in familial susceptibility to AD. ${ }^{8,9}$ APP can be cleaved at different sites to generate a series of fragments, including the $A \beta$ peptides, $\mathrm{N}$-APP and APP intracellular domain (AICD). The extracellular SPs containing the $\mathrm{A} \beta$ peptides have been postulated as a major cause of $A D,{ }^{10,11}$ yet no direct correlation between $\mathrm{A} \beta$ plaques load in the brain of $\mathrm{AD}$ patients and the severity of clinical symptoms has been documented. ${ }^{12}$ On the other hand, $\mathrm{A} \beta$ peptides were also proposed to aggregate into plaques to provide a protective mechanism by sequestering excess $A \beta$ oligomers released from high neuronal activity. ${ }^{13}$ Ectopic expression of APP could induce neuronal cell death, but its mechanism has remained unclear. ${ }^{14} \mathrm{~A}$ recent study suggested that, upon deprivation of trophic factors, APP is cleaved by $\beta$-secretase and an unknown secretase to release N-APP, which binds to the death receptor 6 and induces caspase-dependent neurodegeneration. ${ }^{15}$ AICD was reported to form a transcription complex with Fe65 and histone acetyltransferase Tip60 to regulate gene expression, ${ }^{16}$ and is required for kinesin- mediated axonal transport in vivo. ${ }^{14}$ Despite many studies focusing on the pathological role of $\mathrm{A} \beta$ in neurodegeneration, the function of AICD in development and $A D$ pathogenesis has remained largely elusive.

The forkhead box O (FoxO) transcription factors, initially identified at the chromosomal breakpoints in rhabdomyosarcomas and acute leukemia, ${ }^{17-19}$ contain the forkhead DNA-binding domain that belongs to the O subfamily. ${ }^{20}$ The FoxO proteins have been highly conserved during evolution. Caenorhabditis elegans and Drosophila encode only one FoxO protein, named as Daf- $16^{21}$ and dFoxO, ${ }^{22}$ respectively. Four FoxO proteins, FOXO1, FOXO2, FOXO3a, and FOXO4, have been identified in human, ${ }^{23}$ and an additional member, FOXO6, was reported in mouse. ${ }^{24} \mathrm{FoxO}$ proteins are known to modulate various physiological processes including cell proliferation and differentiation, apoptosis, metabolism, oxidative stress, and lifespan. ${ }^{22,25-27}$ Although studies have confirmed a major role of FoxO in modulating cellular response to oxidative stress, a crucial factor involved in the pathogenesis of $A D$, a direct connection between FoxO proteins and APP, has not been established before.

Drosophila has been previously used as an animal model to study the pathogenesis of $A D$, but most studies have been

\footnotetext{
1Department of Interventional Radiology, Shanghai 10th People's Hospital, Shanghai Key Laboratory of Signaling and Disease Research, School of Life Science and Technology, Tongji University, Shanghai, China and ${ }^{2}$ Department of Anatomy and Neurobiology, School of Medicine, Tongji University, Shanghai, China ${ }^{*}$ Corresponding author: L Xue, Department of Interventional Radiology, Shanghai 10th People's Hospital, Shanghai Key Laboratory for Signaling and Disease Research, School of Life Science and Technology, Tongji University, 1239 Siping Road, Shanghai 200092, China. Tel/Fax: +86 21 65985407; E-mail: lei.xue @ tongji.edu.cn ${ }^{3}$ These authors contributed equally to this work.

Keywords: app; AICD; alzheimer's disease; foxo

Abbreviations: APP, amyloid precursor protein; AD, Alzheimer's disease; $\mathrm{A} \beta$, amyloid $\beta$; AICD, amyloid precursor protein intracellular domain; FoxO, forkhead box 0 ; SP, senile plaques; APLP1, amyloid precursor-like protein 1; APLP2, amyloid precursor-like protein 2; ICD, intracellular domain; CNS, central nervous system Received 11.9.13; revised 04.2.14; accepted 01.4.14; Edited by A Verkhartsky
} 
focused on the neurotoxic effect of $\mathrm{A} \beta$ peptides. In this work, we found that expression of human APP in Drosophila induces AICD-dependent cell death and locomotion defect, both of which were mediated by the transcription factor $\mathrm{dFoxO}$. This mechanism has been conserved in mammalian cells, as AICDs could physically interact with FoxO proteins and act as a transcriptional co-factor to promote FoxOinduced expression of pro-apoptotic gene Bim.

\section{Results and Discussion}

APP induces cell death in Drosophila neuronal and non-neuronal tissues. To investigate the function of APP in animal development, we first expressed human APP (APP695) in the Drosophila nervous system by the panneuronal elav-Gal4 driver (Figure 1b), and observed elevated cell death in the central nervous system (CNS; Figures $1 c, c^{\prime}, d, d^{\prime}$ and $g$ ). Expression of APP in the developing eye driven by GMR-Gal4 (Figure 1h) also induced strong cell death in third-instar larval eye discs (Figures $1 \mathrm{j}$ and $\mathrm{m}$ ) and produced rough eyes with reduced size in the adults (Figure $1 \mathrm{j}^{\prime}$ ), as compared with the control (Figures $1 \mathrm{i}$ and $\mathrm{i}^{\prime}$ ). Collectively, these results indicate that ectopic expression of APP induces strong cell death in the Drosophila nervous system.

To examine whether APP also induces cell death in tissues other than the nervous system, we expressed APP in the developing thorax driven by pnr-Gal4 (Figure 1n). Compared with the control (Figures 10 and $o^{\prime}$ ), expression of APP triggered cell death in the thorax region of third-instar larval wing disc (Figure 1p) and produced a small scutellum phenotype in the adult thorax (dashed line box, Figure 1 $p^{\prime}$ ). A similar scutellum phenotype was also observed when APP expression was driven by ap-Gal4 (Supplementary Figure $\mathrm{S} 1 \mathrm{~b})$. In addition, expression of APP (ptc $>\mathrm{APP}$ ) along the anterior/posterior compartment boundary in the larval wing disc (Figure $2 \mathrm{~m}$ ) induced robust cell death (Figure 20) and produced a loss of the anterior cross vein phenotype in the adult wing (Figure $2 b$ ), as compared with the control (Figures $2 a$ and $n$ ). Furthermore, expression of APP in the developing wing pouch driven by sd-Gal4 $(s d>$ APP) provoked extensive cell death and generated a small wing phenotype in the adults (Supplementary Figure S2b). Together, these results demonstrate that APP is able to induce cell death in non-neuronal tissues during development. a

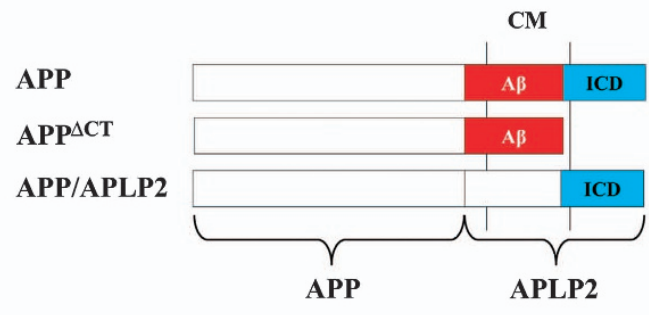

GFP

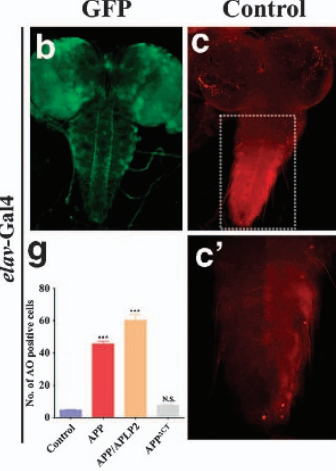

APP

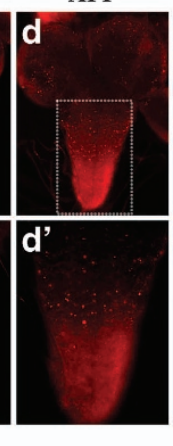

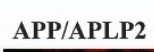

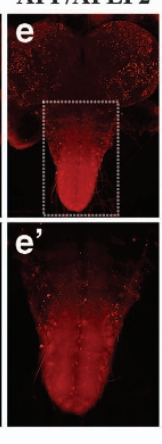

$\mathbf{A P P}^{\Delta C T}$

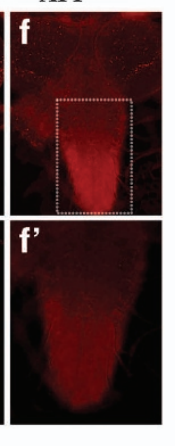

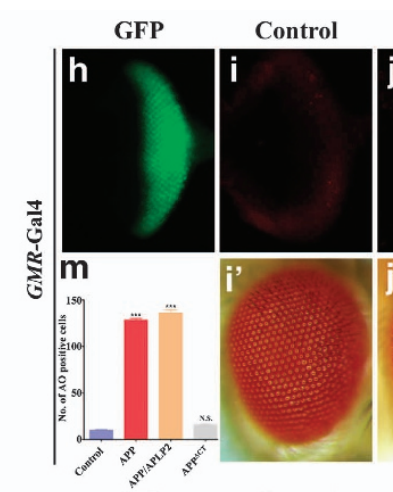

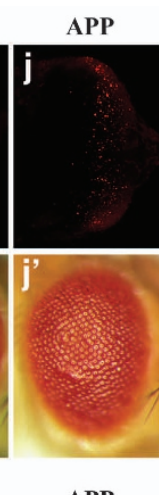

APP/APLP2 $\quad$ APP $^{\triangle C T}$

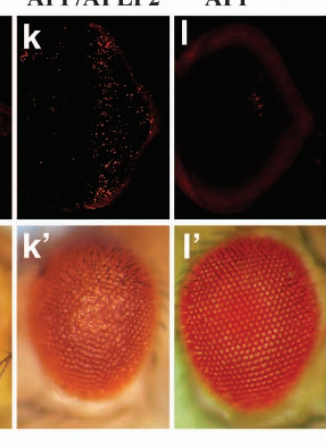

GFP

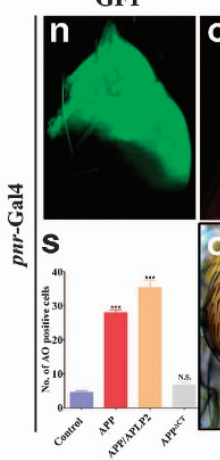

Control

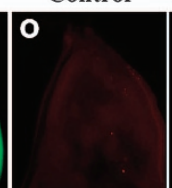

APP

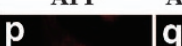

APP/APLP2 APP ${ }^{\triangle C T}$

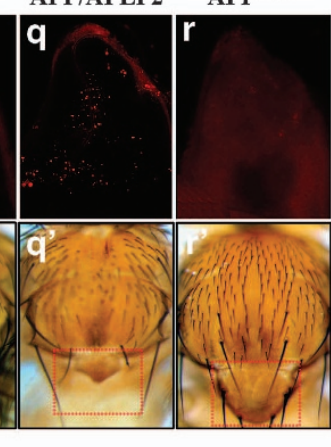

Figure 1 APP induces AICD-dependent cell death in Drosophila. Schematic representation of APP, APP ${ }^{\triangle C T}$, and APP/APLP2 chimeric proteins was shown (a), the A $\beta$ (red) and ICD (blue) domains are indicated. ICD represents the intracellular domain, whereas CM stands for the cell membrane. Fluorescent images of GFP expression (b, $h$ and $\mathbf{n}$ ) or acridine orange staining $\left(\mathbf{c}-\mathbf{f}, \mathbf{c}^{\prime}-\mathbf{f}^{\prime}, \mathbf{i}-\mathbf{I}\right.$, and $\left.\mathbf{0}-\mathbf{r}\right)$ of the central nervous system $(\mathbf{c}-\mathbf{f})$, ventral nerve cord $\left(\mathbf{c}^{\prime}-\mathbf{f}^{\prime}\right)$, eye (i-I), or wing (notum tip, o-r) disc from 3rd instar larvae and light images of adult eye $\left(\mathbf{i}^{\prime}-\mathbf{I}^{\prime}\right)$ or thorax $\left(\mathbf{o}^{\prime}-\mathbf{r}^{\prime}\right)$ are shown. elav-Gal4 $\left(\mathbf{b}-\mathbf{f}, \mathbf{e}^{\prime}-\mathbf{f}^{\prime}\right)$, GMR-Gal4 $\left(\mathbf{h}-\mathbf{I}\right.$ and $\left.\mathbf{i}^{\prime}-\mathbf{I}^{\prime}\right)$, or pnr-Gal4 $\left(\mathbf{n}-\mathbf{r}\right.$ and $\left.\mathbf{o}^{\prime}-\mathbf{r}^{\prime}\right)$ was used to drive the expression of GFP ( $\mathbf{b}, \mathbf{c}, \mathbf{c}^{\prime}, \mathbf{h}, \mathbf{i}, \mathbf{i}^{\prime}, \mathbf{n}, \mathbf{o}$, and $\left.\mathbf{o}^{\prime}\right), \operatorname{APP}\left(\mathbf{d}, \mathbf{d}^{\prime}, \mathbf{j}, \mathbf{j}^{\prime}, \mathbf{p}\right.$, and $\left.\mathbf{p}^{\prime}\right)$, APP/APLP2 $\left(\mathbf{e}, \mathbf{e}^{\prime}, \mathbf{k}, \mathbf{k}^{\prime}, \mathbf{q}\right.$, and $\left.\mathbf{q}^{\prime}\right)$, or APP ${ }^{\Delta C T}\left(\mathbf{f}, \mathbf{f}^{\prime}, \mathbf{l}, \mathbf{l}^{\prime}, \mathbf{r}\right.$ and $\left.\mathbf{r}^{\prime}\right)$. $\mathbf{c}^{\prime}-\mathbf{f}^{\prime}$ are high magnification of boxed areas in $\mathbf{c}-\mathbf{f} . \mathbf{g}, \mathbf{m}$, and $\mathbf{s}$ are statistical analysis of acridine orange-positive cells in $\mathbf{c}-\mathbf{f}, \mathbf{i}-\mathbf{l}$, and $\mathbf{0}-\mathbf{r}$, respectively. Error bars means \pm S.E.M., ${ }^{* \star *} P \leq 0.001$. Genotypes: UAS-GFPI + ; elav-Gal4/ + (b, c , and $\left.\mathbf{c}^{\prime}\right) ;$ UAS-APP/ + ; elav-Gal4/ + (d and $\left.\mathbf{d}^{\prime}\right) ;$ UAS-APP/APLP2/ + ; elav-Gal4/ + (e and $\left.\mathbf{e}^{\prime}\right) ;$ UAS-APP $^{\Delta C T} /+;$ elav-Gal4/ + (f and f');

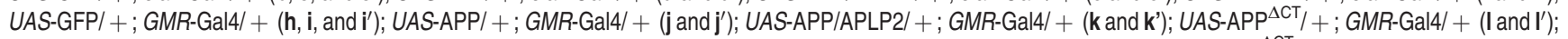
UAS-GFPI + ; pnr-Gal4/ (n, o, and $\left.\mathbf{o}^{\prime}\right) ;$ UAS-APPI +; pnr-Gal4/ + (p and $\left.\mathbf{p}^{\prime}\right) ;$ UAS-APP/APLP2 $/+;$ pnr-Gal4/ + (q and $\left.\mathbf{q}^{\prime}\right) ;$ UAS-APP $^{\Delta C T} /+;$ pnr-Gal4/ + (r and $\left.\mathbf{r}^{\prime}\right)$ 
APP induces cell death through the cleavage of AICD. APP belongs to a conserved family that includes two additional amyloid precursor-like proteins, APLP1 and APLP2, which share with APP the intracellular domain (ICD), but lack the $A \beta$ domain. ${ }^{28,29}$ These three proteins carry out essential overlapping functions in the development, as demonstrated by phenotypes of double or triple knock-out mice. ${ }^{30-33}$ To examine whether $\mathrm{A} \beta$ is required for APP-induced cell death, we expressed an APP/APLP2 chimeric protein containing the ICD domain, but not the $\mathrm{A} \beta$ domain (Figure 1a). This chimeric protein was able to recapitulate APP-induced cell death phenotypes (Figures 1e, $e^{\prime}, g, k, k^{\prime}, m, q, q^{\prime}, s$ and $2 c$ ), implying $A \beta$ is dispensable for APP-triggered cell death. On the other hand, expression of $\mathrm{APP}^{\Delta \mathrm{CT}}$, a truncated form of $\mathrm{APP}$ lacking AICD but retaining the $A \beta$ region (Figure 1a), failed to induce cell death in the CNS (Figures 1f, $f^{\prime}$ and $g$ ), eye (Figure 1l, $\mathrm{I}^{\prime}$ and $\mathrm{m}$ ), thorax (Figures $1 \mathrm{r}, \mathrm{r}^{\prime}$ and $\mathrm{s}$ ), and wing (Figures 2d and p; Supplementary Figure S2e). As this transgene expressed APP ${ }^{\Delta C T}$ at a level comparable to that of APP or APP/APLP2, ${ }^{34,35}$ and two additional APP ${ }^{\Delta \mathrm{CT}}$ transgenes also failed to trigger cell death (Supplementary Figure S3), we concluded that AICD is indispensable for APP-induced cell death in the development. Furthermore, expression of a dominant negative form of Presenillin, ${ }^{36} \mathrm{a}$ major component of the $\gamma$-secretase complex that cleaves AICD from APP, ${ }^{37}$ suppressed APP-induced cell death (Figure $2 \mathrm{e}$ and data not shown), suggesting that APP induces cell death through the cleavage of AICD. Consistent with our finding, AICD, but not $A \beta$, is required for APP-induced blistered-wing phenotype and axonal transport defect. $^{14,35}$

APP functions through dFoxo in Drosophila. To identify the downstream factors that mediate APP-induced cell death, we performed a genetic screen in Drosophila for mutants and UAS-RNAi lines that dominantly suppress APPinduced cell death. We found that ptc $>$ APP-induced cell death in the larval wing disc (Figure 20) and the loss of anterior cross vein phenotype in the adult wing (Figure 2b) were significantly suppressed by mutations in the dfoxO gene (Figures 2f, $\mathrm{k}$, I and q) or expression of two independent dfoxO RNAi (Figures 2g, h, k, I and r; Supplementary Figures $\mathrm{S} 5 \mathrm{~g}$ and $\mathrm{k}$ ). The suppression of cell death is much stronger in heterozygous dfoxo ${ }^{194}$ mutants than by the expression of two dfoxO RNAi (Figures 2q, r and l; Supplementary Figure S5k), as dfoxo mRNA is more significantly reduced in heterozygous dfoxo ${ }^{494}$ mutants (Supplementary Figure S4). dfoxO $\mathrm{O}^{494}$ is a recently generated null allele that removes $>20 \mathrm{~kb}$ of the dfoxO locus and produces no dFoxO protein, and hence, is stronger than all the previously reported dfoxo alleles. ${ }^{38,39}$ Consistently, loss of dfoxO also suppressed ap>APPinduced scutellum phenotype (Supplementary Figures S1C and d) and $s d>$ APP-induced small wing phenotype (Supplementary Figures S2c and d). On the other hand, ectopic expression of dFoxO or its human ortholog FoxO3a was able to phenocopy that of APP (Figures 2i-k and data not shown). Together, these data suggest that dFoxO is a crucial downstream factor that mediates APP-induced cell death in vivo.
To check whether dFoxO is required for APP-induced pathological defect, we examined the larval locomotion behavior. Compared with the control larvae that moved at a speed of about $430 \mu \mathrm{m} / \mathrm{s}$ (Figures 2s and t; Supplementary Video 1), expression of APP in the CNS (Appl>APP) resulted in enhanced cell death (data not shown) and considerably reduced moving ability $(120 \mu \mathrm{m} / \mathrm{s}$, Figures $2 \mathrm{~s}$ and t; Supplementary Video 2). Expression of APP ${ }^{\Delta C T}$ failed to produce such locomotion defect (data not shown), suggesting AICD is essential for the pathological function of APP. We found that APP-induced locomotion defect was significantly suppressed by a mutation in dfoxO $(330 \mu \mathrm{m} / \mathrm{s}$, Figures 2s and t; Supplementary Video 3) or expression of a dfoxO RNAi $(270 \mu \mathrm{m} / \mathrm{s}$, Figures 2s and t), which by themselves had no obvious effect on locomotion (Figures $2 s$ and $\mathrm{t}$ ). Together, these results indicate that dFoxO is physiologically required for APP-induced behavioral defects.

To further verify the activation of dFoxO by APP in vivo, we examined the expression of pro-apoptotic gene hid, a wellknown transcriptional target of dFoxO. ${ }^{40}$ We found that APP expression driven by $s d-G a l 4$ in the wing pouch resulted in strong activation of hid transcription, as monitored by a hid-lac Z reporter, and reduced wing pouch size (Figures $2 u$ and $v$ ). Both phenotypes were considerably suppressed in dfoxo heterozygous mutant discs (Figure $2 \mathrm{w}$ ), indicating APPinduced hid activation is mediated by dFoxO. Consistently, expression of APP/APLP2 chimera (Supplementary Figure $\mathrm{S6c}$ ), but not that of $\mathrm{APP}^{\triangle \mathrm{CT}}$ (Figure $2 \mathrm{x}$ ), also induced hid transcription, confirming that the ICD domain, but not $\mathrm{A} \beta$, is essential for APP-induced FoxO activation.

AICD physically interacts with FoxO proteins in human cells. Our genetic work in Drosophila suggested that APP induces FoxO-mediated cell death through the release of AICD. To investigate the mechanism by which AICD promotes FoxO-mediate cell death, we co-expressed Myctagged AICD (AICD-Myc) with human FoxO1, FoxO3a, or FoxO4 in HEK 293T cells, and followed with co-immunoprecipitation assay. AICD was able to co-precipitate with FoxO1, FoxO3a, or FoxO4 (Figure 3a), and vice versa, FoxO3a or FoxO4 co-precipitated with AICD (Figure 3b), suggesting that AICD physically interacts with FoxO proteins in vivo. Consistently, AICD co-localized with FoxO predominantly in the cytoplasm of 293T cells (Figure 3e and Supplementary Figure S7). Treatment of cells with $\mathrm{H}_{2} \mathrm{O}_{2}$ induced oxidative stress that promoted translocation of FoxO from the cytoplasm into the nucleus ${ }^{41}$ (Figure $3 f$ and Supplementary Figure S7). AICD also translocated into the nucleus under oxidative stress (Figures $3 c$ and $f$, Supplementary Figure S7), presumably through its interaction with FoxO proteins. In the absence of exogenous FoxO, AICD largely remained in the cytoplasm despite $\mathrm{H}_{2} \mathrm{O}_{2}$ treatment (Figure 3c). Furthermore, AICD was able to translocate into the nucleus with FoxO4A3, a constitutive active form of FoxO4 that localized predominantly in the nucleus, ${ }^{42}$ without oxidative stress challenge (Figures $3 d$ and i). Similar results for AICD and FoxO3a were also obtained in human neuro-blastoma SY-SH5Y cells (Figures $3 \mathrm{~g}$ and $\mathrm{h}$ ). On the other hand, the predominant localization 
of FoxO1 and FoxO3a in the cytoplasm and FoxO4A3 in the nucleus was not affected by the presence of AICD (Supplementary Figure S8).
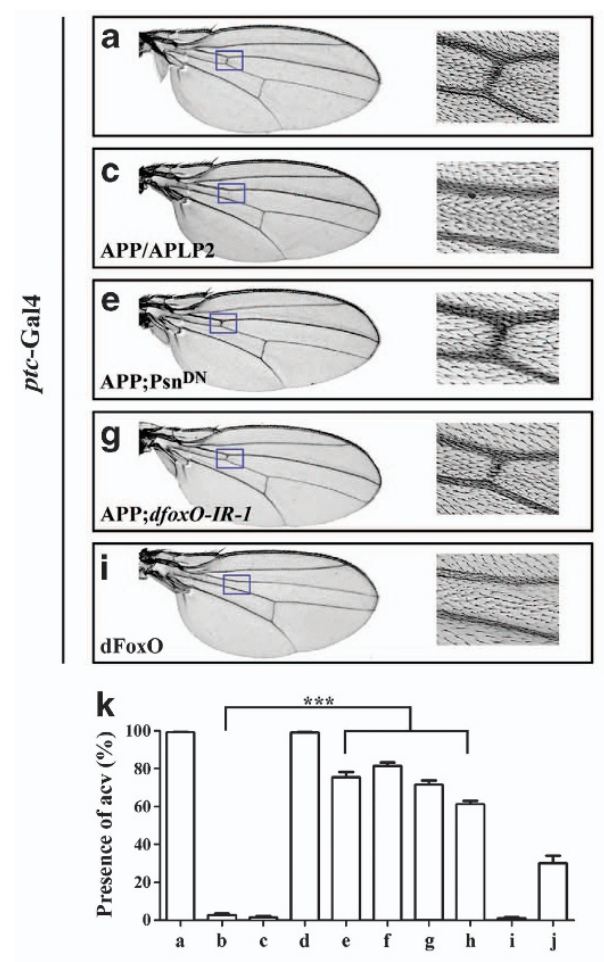

GFP
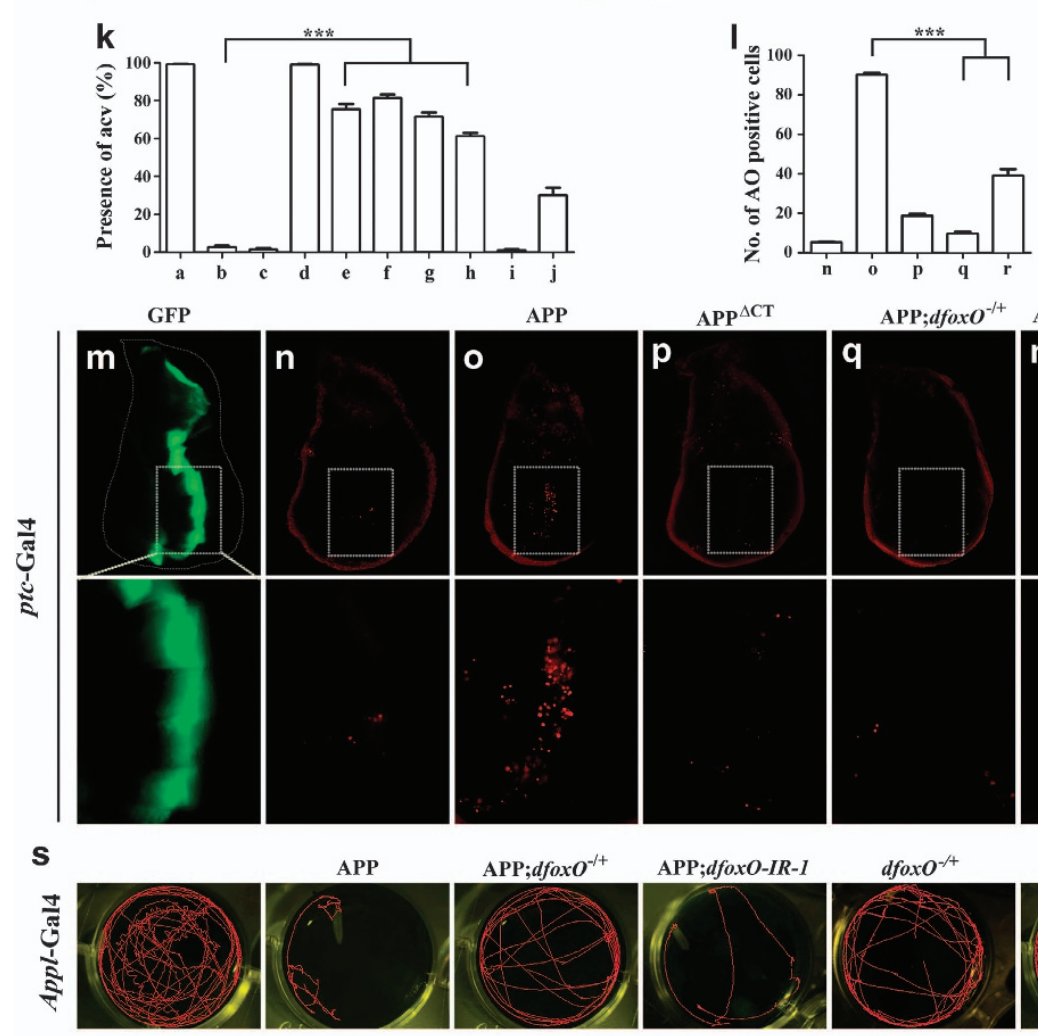

APP; dfoxO-IR-1
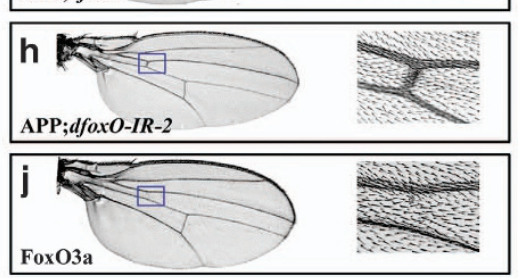

AICD is a transcriptional cofactor of FoxO proteins. Based upon the above results, we hypothesized that AICD could physically interact with FoxO proteins in the cytoplasm,
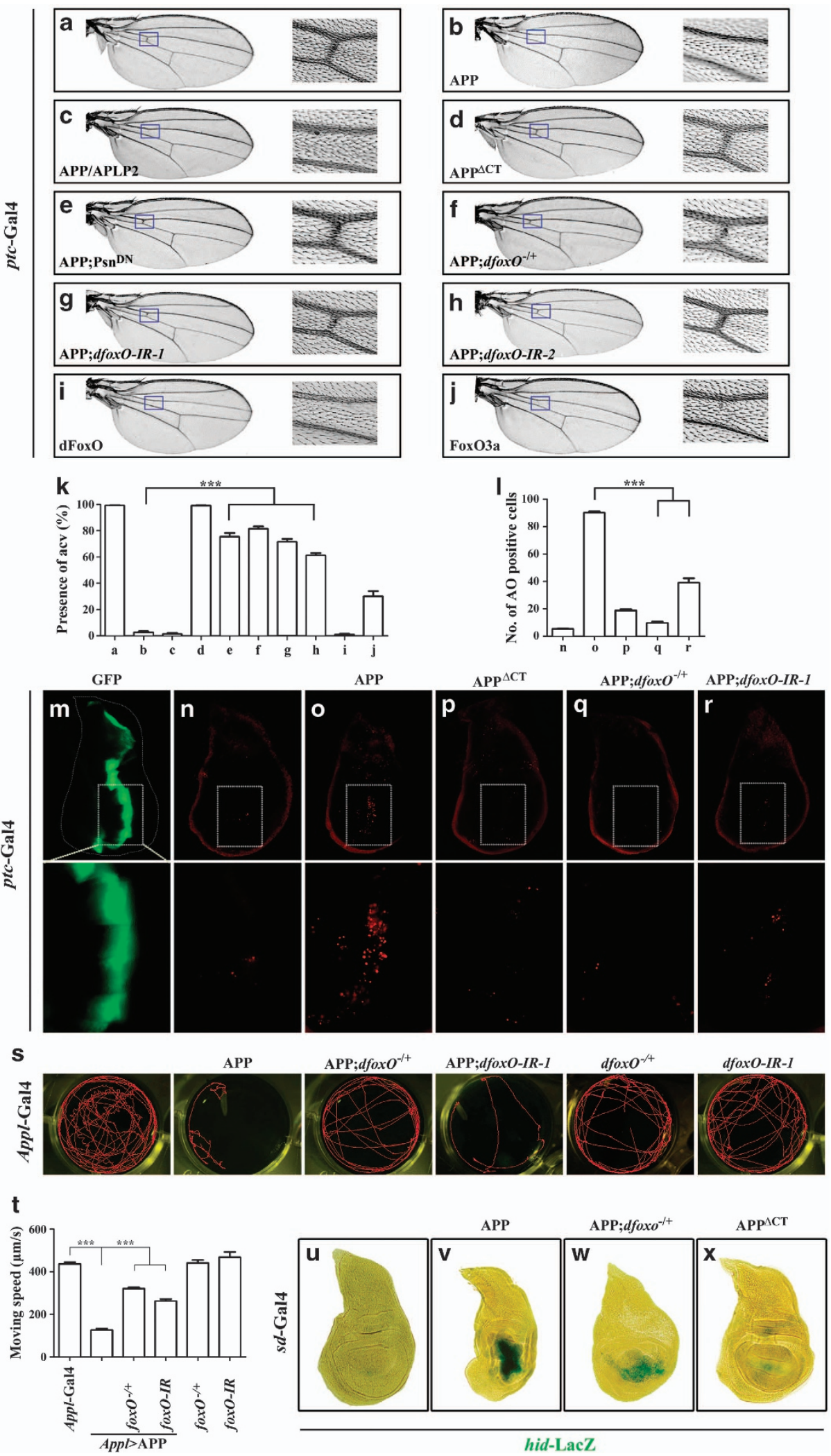
a

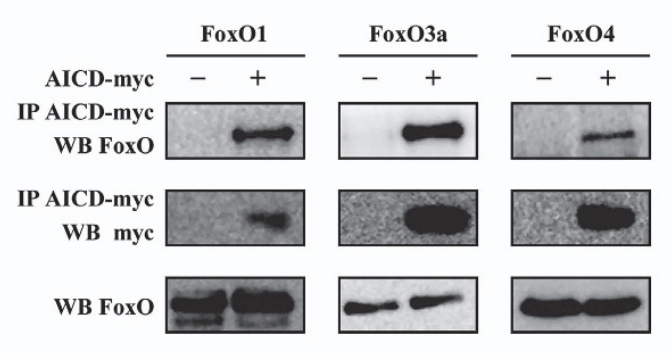

b

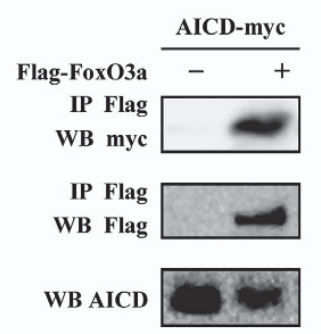

C

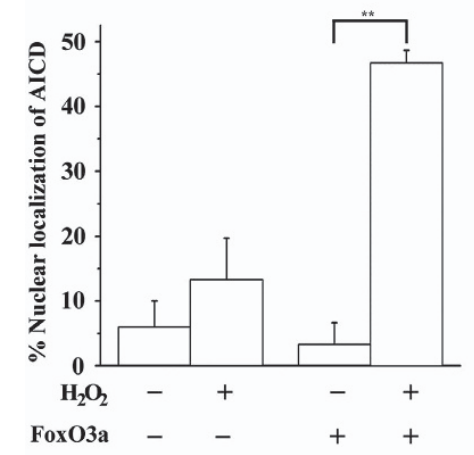

AICD-myc
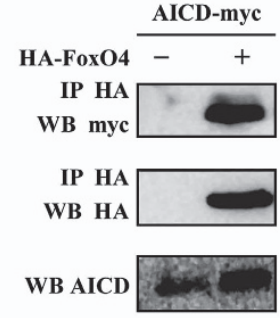

d

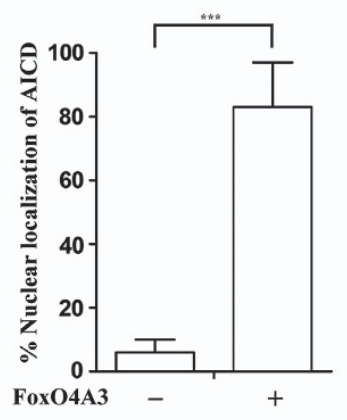

FoxO3a-GFP
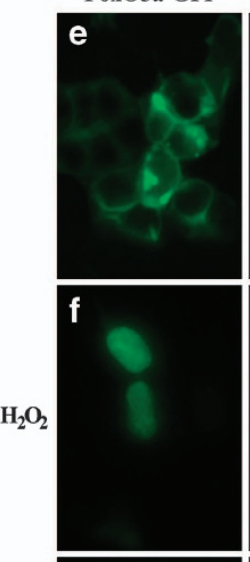

AICD
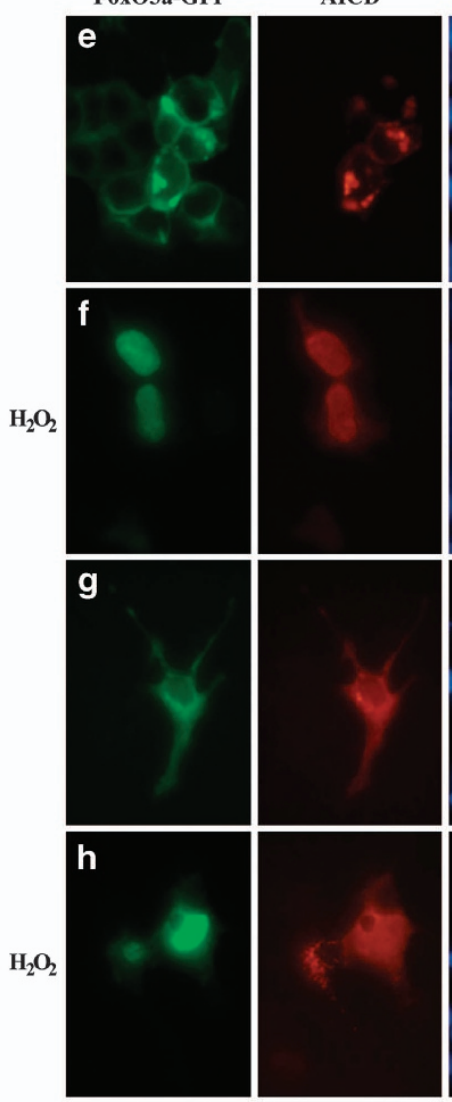

FoxO4A3-GFP

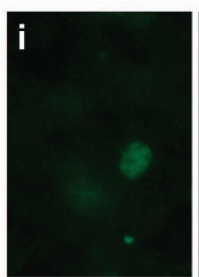

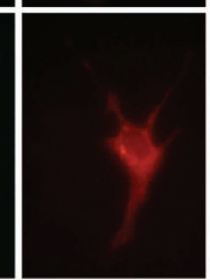

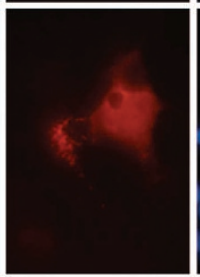

AICD

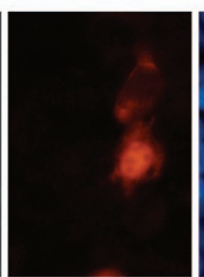

Hoechst
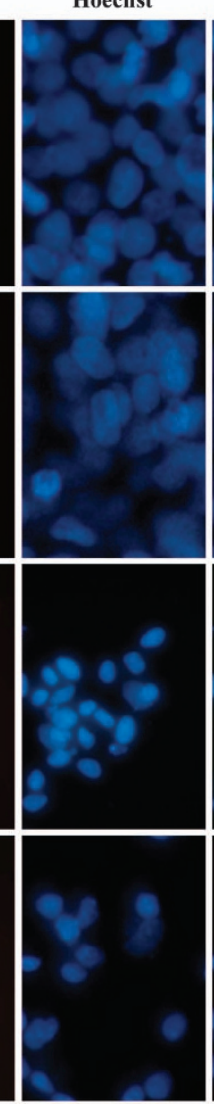

Hoechst

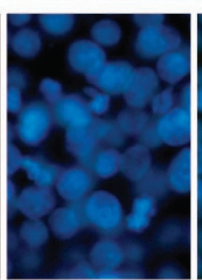

Merge
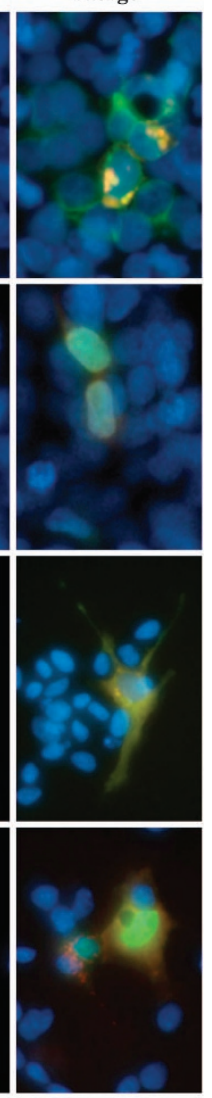

Merge

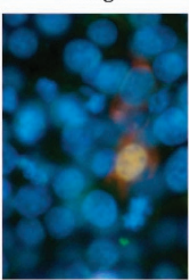

Figure 3 Physical interaction between AICD and FoxO in human cells. (a) Fox01, FoxO3a, or FoxO4 co-precipitated with AICD in HEK 293T cells. (b) AICD co-precipitated with FoxO3a or FoxO4 in HEK 293T cells. (c) FoxO3a enriched AICD nuclear accumulation in 293T cells under oxidative stress. A minimum of 200 cells per condition were counted. ${ }^{\star \star} P \leq 0.01$. (d) FoxO4A3 enriched AICD nuclear accumulation in $293 \mathrm{~T}$ cells without oxidative stress. A minimum of 200 cells per condition were counted. ${ }^{* \star} P \leq 0.001$. (e-h) AICD co-localized with FoxO3a in 293T (e and f) or SY-SH5Y cells ( $\mathbf{g}$ and $\mathbf{h}$ ) before (e and $\mathbf{g}$ ) or after ( $\mathbf{f}$ and $\mathbf{h}$ ) $\mathrm{H}_{2} \mathrm{O}_{2}$ treatment. AICD co-localized with FoxO4A3 in HEK 293T cells without oxidative stress (i). Cells were transfected with AICD-Myc and FoxO4A3 in HEK 293T cells, stained with Hoechst and incubated with anti-Myc and anti-HA antibodies. Immunoreactivity was detected with IgG conjugated to Alexa Fluor 546 (red, detects AICD), IgG conjugated to Alexa Fluor 488 (green, detects FoxO4A3). Co-localization was visualized by fluorescence microscope

Figure 2 dFoxO mediates APP-induced cell death and locomotion defect in Drosophila (a-j), light images of adult wing are shown. The areas for anterior cross vein (ACV) are boxed on the left and enlarged on the right. ptc-Gal4 was used to drive the expression of GFP (a), APP (b,e-h), APP/APLP2 (c), APP ${ }^{\triangle C T}(\mathbf{d}), d_{F o x O}(\mathbf{i})$, or human FoxO3a (j). ptc $>$ APP induced loss-of-ACV phenotype (b) was suppressed by the expression of a dominant negative Psn (e), mutation (f), or RNAi downregulation ( $\mathbf{g}, \mathbf{h}$ ) of dfoxO. (k) Statistical analysis of the ACV phenotype of given genotype in a-j. The percentage of adult wings with the presence of ACV is shown. Error bars means \pm S.E.M., ${ }^{* \star \star} P \leq 0.001$. (I) Statistical analysis of acridine orange-positive cells in $\mathbf{n}-\mathrm{r}$. Error bars means \pm S.E.M., ${ }^{* \star *} P \leq 0.001$. ( $\mathbf{m}-\mathbf{r}$ ) Fluorescent images of GFP expression (m) or acridine orange staining $(\mathbf{n}-\mathbf{r})$ of wing disc from 3rd instar larvae are shown. Lower panels are high magnification of boxed areas in the upper panels. ptc-Gal4 was used to drive the expression of $\operatorname{GFP}(\mathbf{m}, \mathbf{n}), \operatorname{APP}(\mathbf{o}, \mathbf{q}$, and $\mathbf{r})$ or $\operatorname{APP}^{\Delta C T}(\mathbf{p})$. APP-induced cell death was suppressed by mutation (q) or RNAi downregulation (r) of dfoxO. (s) The larval moving track was recorded. APP expression driven by Appl-Gal4 resulted in larval locomotion defect, which was suppressed by mutation or RNAi downregulation of dfoxO. Moving speed was shown in t. Error bars means \pm S.E.M., ${ }^{* * *} P \leq 0.001$. (u-x) X-Gal staining of a hid-LacZ reporter gene in 3rd instar wing discs. $s d-G a l 4$ was used to drive the expression of GFP $(\mathbf{u}), \operatorname{APP}(\mathbf{v}, \mathbf{w}), \operatorname{APP}^{\Delta C T}(\mathbf{v}), \operatorname{APP}$ induced hid activation was partially suppressed in dfoxO heterozygous mutants (w). Genotypes: ptc-Gal4/UAS-GFP (a, m, and n); ptc-Gal4 UAS-APP/+ (b and o); ptc-Gal4/+; UAS-APP/APLP2/ + (c); ptc-Gal4/UAS-APP ${ }^{\Delta C T}$ (d and p); ptc-Gal4 UAS-APP/

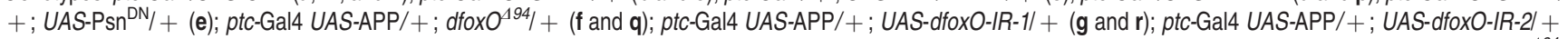
(h); ptc-Gal4/UAS-dFoxO (i); ptc-Gal4/UAS-FoxO3a (j); Appl-Gal4/ + ; UAS-GFPI + (left); Appl-Gal4/ + ; UAS-APPI + (2nd to left); Appl-Gal4/ + ; UAS-APP/ + ; dfoxO ${ }^{494 /}$ + (3rd to left); Appl-Gal4/ + ; UAS-APPI + ; UAS-dfoxO-IR-1/ + (4th to left); dfoxO ${ }^{294 /}+$ (5th to left) and UAS-dfoxO-IR-1/ + (right) (s and t); sd-Gal4/ + ; UAS-GFP/ + ;

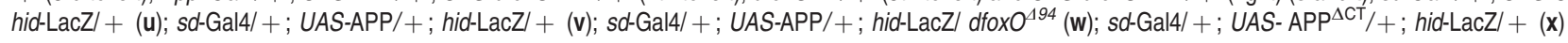


translocate with FoxO into the nucleus under oxidative stress, and function as a transcriptional co-factor of FoxO. To test this hypothesis, we examine the effect of AICD on the transcription of FoxO target genes. The pro-apoptotic gene $\mathrm{Bim}$, which encodes a $\mathrm{BH} 3-$ only protein that directly activates the cell death machinery, is a well-known transcriptional target of FoxO proteins. ${ }^{43}$ In consistent with the hypothesis, AICD was able to upregulate Bim promoter-mediated transcription (Figure 4a), possibly through its interaction with the endogenous FoxO proteins. Furthermore, AICD cooperated with the constitutive active FoxO4A3 to synergistically activate luciferase expression driven by Bim promoter (Figure 4b) or $6 \times$ DBE (Figure 4c), a synthetic promoter containing six FoxO-responsive elements. ${ }^{44}$ A similar cooperative effect with FoxO4A3 was observed for APPswe or
C99 (Supplementary Figure S9) that comprises AICD, or the $\gamma$-secretase PS1 (Supplementary Figure S10) that promotes AICD production from endogenous APP. Hydrogen peroxide treatment of cells stably expressing C99-GFP ${ }^{45}$ resulted in enhanced AICD production accompanied with elevated Bim expression (lane 2, Figure 4d). On the other hand, AICD production was compromised by two $\gamma$-secretase inhibitors, DBZ and Comp E, which resulted in significantly reduced Bim expression (lane 4 and 5, Figure 4d). Together, these data suggested that AICD acts as a transcriptional co-factor of FoxO to activate the expression of apoptotic gene Bim. Finally, consistent with our hypothesis, Bim expression was upregulated in the frontal lobe of APP/PS1 double transgenic mice (Supplementary Figure S11), and in the entorhinal cortical neurons of $A D$ patient brains. ${ }^{46}$

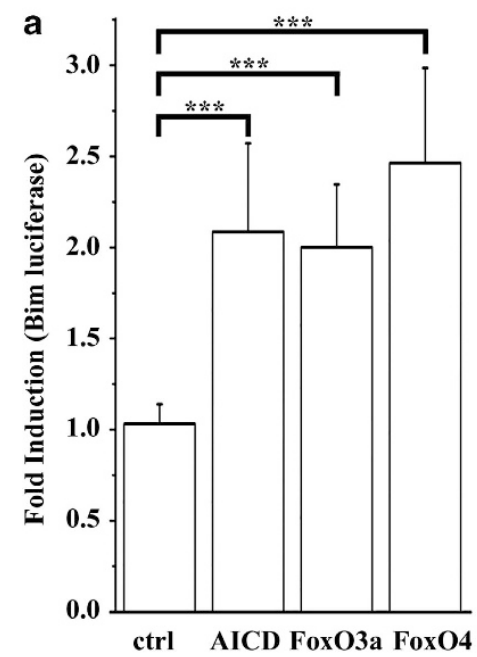

b
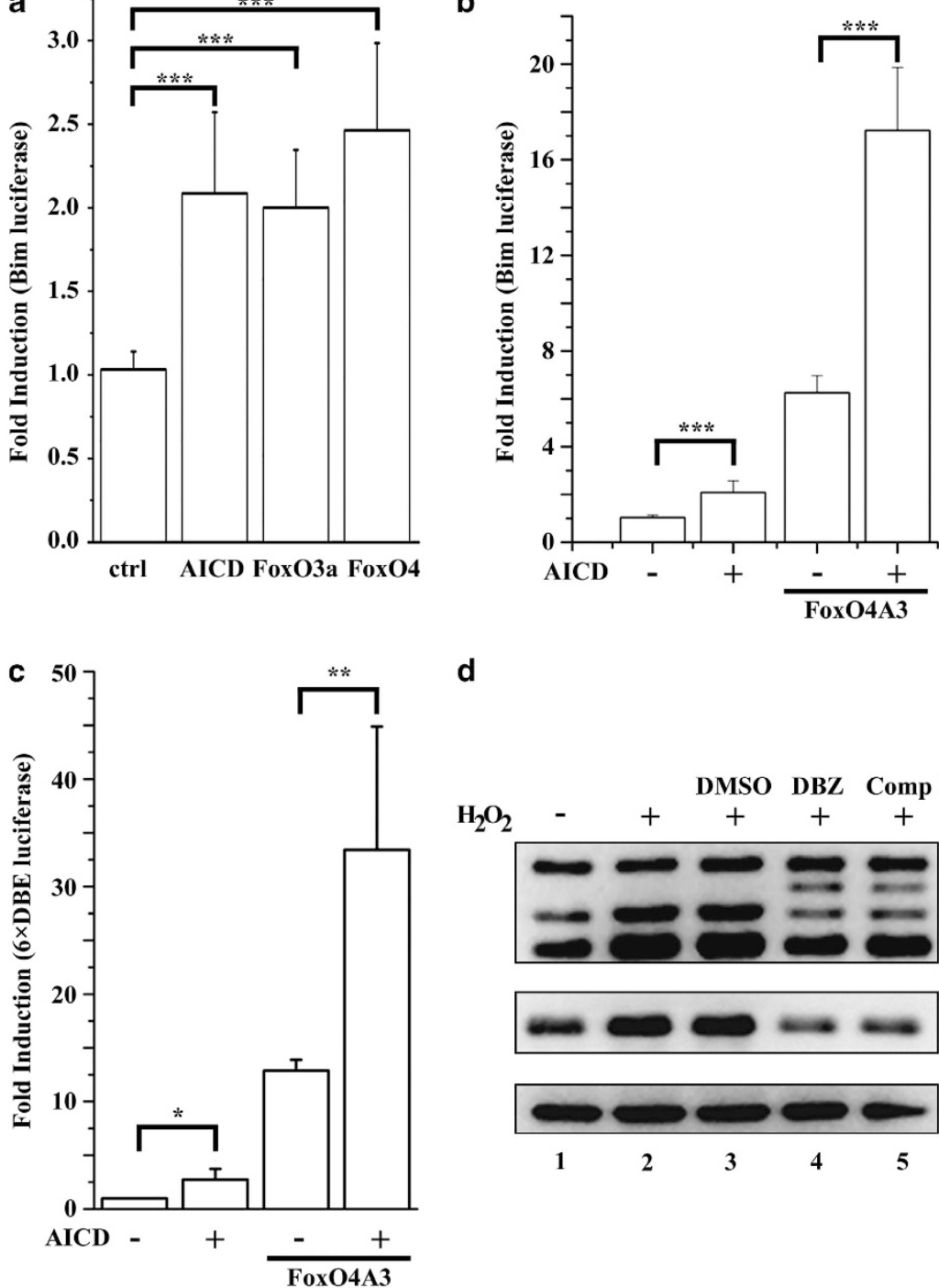

d
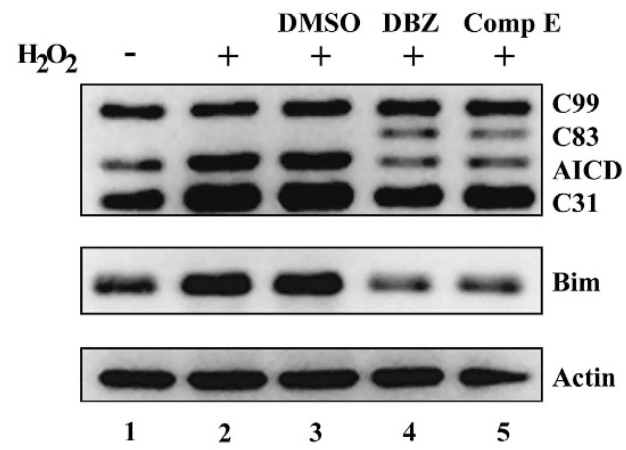

Figure 4 AICD promotes the transcription of FoxO target genes. (a) Expression of AICD-, FoxO3a-, or FoxO4-induced Bim promoter-directed luciferase expression in HEK 293T cells. (b and c) AICD cooperated with FoxO4A3 to stimulate luciferase expression driven by Bim promoter (b) or $6 \times \mathrm{DBE}(\mathbf{c}) .{ }^{*} P \leq 0.05,{ }^{* \star} P \leq 0.01,{ }^{* \star \star} P \leq 0.001$. (d) Elevated AICD production (top) and Bim expression (middle) upon $\mathrm{H}_{2} \mathrm{O}_{2}$ challenge (lane 2, $200 \mu \mathrm{M}$ for $12 \mathrm{~h}$ ) in 293T cells stably expressing C99-EGFP (lane 1) were downregulated by pre-treatment ( $2 \mu \mathrm{M}$ for $3 \mathrm{~h}$ ) with $\gamma$-secretase inhibitor DBZ (lane 4 ) or Compound $\mathrm{E}$ (lane 5 ), but not by that of DMSO (lane 3 ). Actin was served as a loading control (bottom) 


\section{Conclusion}

$A D$ is one of the most prevalent neurodegenerative diseases in the world, although its underlying mechanism has remained largely elusive. In the present study, we analyzed the pathological functions of APP, an AD causative gene, in Drosophila, mouse and human cells. We demonstrated that ectopic expression of APP induces cell death and locomotion defect in Drosophila. Such function of APP depends on the production of AICD, and is mediated by the transcription factor dFoxO. Further studies in human cells confirmed that AICD interacts and co-localizes with FoxO proteins. Upon oxidative stress, AICD translocates from the cytoplasm into the nucleus together with FoxO, and functions as a transcriptional co-factor of FoxO to induce the transcription of Bim, a pro-apoptotic gene that activates the cell death machinery. Our findings not only expand the existing body of knowledge about the physiological function of APP, but also shed light on discovering a potential cure for AD.

\section{Materials and Methods}

Fly Stocks. Drosophila strains used include: UAS-APP, UAS-APP $695^{\triangle C T}$, and UAS-APP/APLP2, ${ }^{34}$ UAS-dFoxO and UAS-FoxO3a, ${ }^{22}$ UAS-Psn ${ }^{\mathrm{DN}},{ }^{36} \mathrm{dFoxO} \mathrm{O}^{194}, 38$ Appl-Gal4, ${ }^{47}$ elav-Gal4, GMR-Gal4, ptc-Gal4, hid-LacZ, pnr-Gal4, and sd-Gal4, ${ }^{48}$ ap-Gal4, ${ }^{49}$ UAS-GFP, ${ }^{50}$ UAS-dFoxo-IR-1 and UAS-dFoxo-IR- ${ }^{39}$ were previously described. Two additional UAS-APP695 ${ }^{\Delta C T}$ transgenic lines (\#2 and \#3) were obtained from the Bloomington Drosophila Stock Center at Indiana University (Bloomington, IN, USA) (\#29875 and \#29876).

Acridine orange staining. Brain, eye, and wing discs were dissected from the 3rd instar larvae in PBST and stained for acridine orange as described. ${ }^{50}$

X-Gal staining. Wing discs were dissected from 3rd instar larvae in PBST and stained for $\beta$-galactosidase activity as described. ${ }^{51}$

Larva locomotion test. Third instar larvae were collected and rinsed in 1\% PBS, and subsequently transferred onto $3 \%$ agarose plate. The larvae were allowed to rest for $5 \mathrm{~min}$ before videotaping. The locomotion behaviors were analyzed by the Nikon software (NIS-Elements D, Nikon, Shanghai, China). Each genotype was tested with five larvae and each larva was tested for five times. For the track of the larvae, each genotype was recorded for $10 \mathrm{~min}$ and repeated for five times.

Plasmids. Expression vectors for GFP-FOXO1, GFP-FOXO3a, Flag-FOXO3a, and Bim-luciferase reporter were obtained from Z Yuan; ${ }^{52}$ HA-FoxO4, HAFoxO4A3, and $6 \times$ DBE-luciferase were obtained from B Burgering; ${ }^{44}$ AICD-myc were obtained from $Y$ Zhang; ${ }^{53}$ APPswe and $C 99$ were obtained from $\mathrm{N}$ Jing. ${ }^{45}$

Cells and reagents. HEK 293T cells were maintained in DMEM supplemented with $10 \%$ heat-inactivated fetal bovine serum (FBS). SH-SY5Y cells were grown in RPMI-1640 supplemented with 10\% heat-inactivated FBS. Transfection was performed using FuGENE HD Transfection Reagent (Roche Applied Science, Indianapolis, IN, USA) in accordance with the manufacturer's instructions or calcium-phosphate precipitation by standard procedure. To generate cell lines stably expressing C99-GFP, HEK293 cells were transfected with the construct and selected in the presence of G418 (500 $\mu \mathrm{g} / \mathrm{ml})$. HEK 293 C99-GFP cells were maintained in the medium containing $200 \mu \mathrm{g} / \mathrm{ml}$ of $\mathrm{G} 418$. Antibodies used were: anti-HA, anti-Myc, anti-FLAG, anti-APP, anti-actin, antiGFP, EZview TM Red Anti-HA, anti-Flag M2 Affinity Gel, and protein G-Sepharose beads from Sigma (St. Louis, MO, USA); anti-FOXO1, anti-FOXO3a, anti-FOXO4, and anti-Bim from Cell Signaling (Danvers, MA, USA); Alexa Fluor-546Conjugated Goat Anti-Mouse Antibody and Alexa Fluor-488-conjugated goat anti-rabbit antibody from Molecular Probes (Invitrogen, Carlsbad, CA, USA). The inhibitors of presenilin/ $\gamma$-secretase DBZ and Compound E (Calbiochem, San Diego, CA, USA) were used at $2 \mu \mathrm{M}$ (final concentration).

Immunofluorescence. Cells were transfected with the indicated plasmids for $24 \mathrm{~h}$ and then left untreated or treated with $\mathrm{H}_{2} \mathrm{O}_{2}(200 \mu \mathrm{M}$, final concentration) for $1 \mathrm{~h}$, fixed in $3.7 \%$ formaldehyde and permeabilized with $0.2 \%$ Triton $\mathrm{X}-100$. Cells were incubated with the indicated primary antibodies followed by Alexa Fluor-conjugated secondary antibodies and observed under a fluorescent microscope (Olympus, Tokyo, Japan).

Immunoprecipitation and immunoblotting. Cells were transfected with the indicated plasmids for $24 \mathrm{~h}$. Precleared lysates were incubated with the indicated antibodies followed by precipitation with protein G-Sepharose beads. Immune complexes were washed with lysis buffer, eluted in $2 \times$ SDS sample buffer, and subjected to western blot analysis using corresponding antibodies.

Luciferase reporter assay. HEK 293T cells were transfected with effector plasmids and luciferase reporters. pRL-TK Renilla luciferase construct was co-transfected as an internal control. Cells were harvested and subjected to dual-luciferase assay (Promega, Madison, WI, USA) after $24 \mathrm{~h}$.

\section{Conflict of Interest}

The authors declare no conflict of interest.

Acknowledgements. We thank E Hafen, L Partridge, Bloomington, NIG, and VDRC stock centers for fly stocks, Z Yuan, B Burgering, Y Zhang, and N Jing for plasmids, members of Xue lab for discussion and comments. This work was supported by the National Basic Research Program of China (973 Program; Grant No. 2010CB944901 and 2011CB943903), National Natural Science Foundation of China (Grant No. 31071294, 31171413, and 31371490), the PhD Programs Foundation of Ministry of Education of China (Grant No. 20120072120030), and Shanghai Committee of Science and Technology (Grant No. 09DZ2260100).

1. Pung L, Wang X, Li M, Xue L. The role of APP in Alzheimer's disease. Advan Alzheimer's Dis 2013; 2: 60-65.

2. DeKosky ST, Scheff SW. Synapse loss in frontal cortex biopsies in Alzheimer's disease: correlation with cognitive severity. Ann Neurol 1990; 27: 457-464.

3. Bouras C, Hof PR, Giannakopoulos P, Michel JP, Morrison JH. Regional distribution of neurofibrillary tangles and senile plaques in the cerebral cortex of elderly patients: a quantitative evaluation of a one-year autopsy population from a geriatric hospital. Cereb Cortex 1994; 4: 138-150.

4. Trojanowski JQ, Lee VM. Phosphorylation of neuronal cytoskeletal proteins in Alzheimer's disease and Lewy body dementias. Ann N Y Acad Sci 1994; 747: 92-109.

5. Goedert M, Sisodia SS, Price DL. Neurofibrillary tangles and beta-amyloid deposits in Alzheimer's disease. Curr Opin Neurobiol 1991; 1: 441-447.

6. Alzheimer A, Stelzmann RA, Schnitzlein HN, Murtagh FR. An English translation of Alzheimer's 1907 paper, "Uber eine eigenartige Erkankung der Himrinde". Clin Anat 1995; 8: 429-431.

7. Kang J, Lemaire HG, Unterbeck A, Salbaum JM, Masters CL, Grzeschik KH et al. The precursor of Alzheimer's disease amyloid A4 protein resembles a cell-surface receptor. Nature 1987; 325: 733-736.

8. Goate A, Chartier-Harlin MC, Mullan M, Brown J, Crawford F, Fidani L et al. Segregation of a missense mutation in the amyloid precursor protein gene with familial Alzheimer's disease. Nature 1991; 349: 704-706.

9. Karlinsky H, Vaula G, Haines JL, Ridgley J, Bergeron C, Mortilla M et al. Molecular and prospective phenotypic characterization of a pedigree with familial Alzheimer's disease and a missense mutation in codon 717 of the beta-amyloid precursor protein gene. Neurology 1992; 42: 1445-1453.

10. Tanzi RE, Bertram L. Twenty years of the Alzheimer's disease amyloid hypothesis: a genetic perspective. Cell 2005; 120: 545-555.

11. Kim D, Tsai LH. Bridging physiology and pathology in AD. Cell 2009; 137: 997-1000.

12. Arriagada PV, Growdon JH, Hedley-Whyte ET, Hyman BT. Neurofibrillary tangles but not senile plaques parallel duration and severity of Alzheimer's disease. Neurology 1992; 42: 631-639.

13. Cuajungco MP, Goldstein LE, Nunomura A, Smith MA, Lim JT, Atwood CS et al. Evidence that the beta-amyloid plaques of Alzheimer's disease represent the redox-silencing and entombment of abeta by zinc. J Biol Chem 2000; 275: 19439-19442.

14. Gunawardena S, Goldstein LS. Disruption of axonal transport and neuronal viability by amyloid precursor protein mutations in Drosophila. Neuron 2001; 32: 389-401.

15. Nikolaev A, McLaughlin T, O'Leary DD, Tessier-Lavigne M. APP binds DR6 to trigger axon pruning and neuron death via distinct caspases. Nature 2009; 457: 981-989.

16. Cao X, Sudhof TC. A transcriptionally [correction of transcriptively] active complex of APP with Fe65 and histone acetyltransferase Tip60. Science 2001; 293: 115-120.

17. Borkhardt A, Repp R, Haas OA, Leis T, Harbott J, Kreuder J et al. Cloning and characterization of AFX, the gene that fuses to MLL in acute leukemias with a $\mathrm{t}(\mathrm{X} ; 11)(q 13 ; q 23)$. Oncogene 1997; 14: 195-202. 
18. Galili N, Davis RJ, Fredericks WJ, Mukhopadhyay S, Rauscher 3rd FJ, Emanuel BS et al. Fusion of a fork head domain gene to PAX3 in the solid tumour alveolar rhabdomyosarcoma. Nature genetics 1993; 5: 230-235.

19. Hillion J, Le Coniat M, Jonveaux P, Berger R, Bernard OA. AF6q21, a novel partner of the MLL gene in $t(6 ; 11)(q 21 ; q 23)$, defines a forkhead transcriptional factor subfamily. Blood 1997: 90: 3714-3719.

20. Kaestner KH, Knochel W, Martinez DE. Unified nomenclature for the winged helix/forkhead transcription factors. Genes Dev 2000; 14: 142-146.

21. Lin K, Dorman JB, Rodan A, Kenyon C. daf-16: An HNF-3/forkhead family member that can function to double the life-span of Caenorhabditis elegans. Science 1997; 278: 1319-1322.

22. Junger MA, Rintelen $F$, Stocker $H$, Wasserman JD, Végh $M$, Radimerski $T$ et al. The Drosophila forkhead transcription factor FOXO mediates the reduction in cell number associated with reduced insulin signaling. J Biol 2003; 2: 20.

23. Birkenkamp KU, Coffer PJ. Regulation of cell survival and proliferation by the FOXO (Forkhead box, class O) subfamily of Forkhead transcription factors. Biochem Soc Trans 2003; 31: 292-297.

24. Jacobs FM, van der Heide LP, Wijchers PJ, Burbach JP, Hoekman MF et al. FoxO6, a novel member of the FoxO class of transcription factors with distinct shuttling dynamics. J Biol Chem 2003; 278: 35959-35967.

25. Puig O, Marr MT, Ruhf ML, Tjian R. Control of cell number by Drosophila FOXO: downstream and feedback regulation of the insulin receptor pathway. Genes Dev2003; 17 2006-2020.

26. Giannakou ME, Goss M, Jünger MA, Hafen E, Leevers SJ, Partridge $L$ et al. Long-lived Drosophila with overexpressed dFOXO in adult fat body. Science 2004; 305: 361.

27. Hwangbo DS, Gershman B, Tu MP, Palmer M, Tatar M. Drosophila dFOXO controls lifespan and regulates insulin signalling in brain and fat body. Nature 2004; 429: 562-566.

28. Anliker B, Muller $U$. The functions of mammalian amyloid precursor protein and related amyloid precursor-like proteins. Neuro-degener Dis 2006; 3: 239-246.

29. Muller UC, Zheng H. Physiological functions of APP family proteins. Cold Spring Harb Perspect Med 2012; 2: a006288.

30. Heber S, Herms J, Gajic V, Hainfellner J, Aguzzi A, Rülicke T et al. Mice with combined gene knock-outs reveal essential and partially redundant functions of amyloid precursor protein family members. J Neurosci 2000; 20: 7951-7963.

31. Herms J, Anliker B, Heber S, Ring S, Fuhrmann M, Kretzschmar $\mathrm{H}$ et al. Cortical dysplasia resembling human type 2 lissencephaly in mice lacking all three APP family members. EMBO J 2004; 23: 4106-4115.

32. von Koch CS, Zheng H, Chen H, Trumbauer M, Thinakaran G, van der Ploeg LH et al. Generation of APLP2 KO mice and early postnatal lethality in APLP2/APP double KO mice. Neurobiol Aging 1997; 18: 661-669.

33. Wang P, Yang G, Mosier DR, Chang P, Zaidi T, Gong YD et al. Defective neuromuscular synapses in mice lacking amyloid precursor protein (APP) and APP-Like protein 2 J Neurosci 2005; 25: 1219-1225.

34. Fossgreen A, Brückner B, Czech C, Masters CL, Beyreuther K, Paro R et al. Transgenic Drosophila expressing human amyloid precursor protein show gamma-secretase activity and a blistered-wing phenotype. Proc Natl Acad Sci USA 1998; 95: 13703-13708.

35. Merdes G, Soba P, Loewer A, Bilic MV, Beyreuther K, Paro R et al. Interference of human and Drosophila APP and APP-like proteins with PNS development in Drosophila. EMBO J 2004; 23: 4082-4095

36. Carmine-Simmen K, Proctor T, Tschäpe J, Poeck B, Triphan T, Strauss R et al. Neurotoxic effects induced by the Drosophila amyloid-beta peptide suggest a conserved toxic function. Neurobiol Dis 2009; 33: 274-281.

37. Passer B, Pellegrini L, Russo C, Siegel RM, Lenardo MJ, Schettini G et al. Generation of an apoptotic intracellular peptide by gamma-secretase cleavage of Alzheimer's amyloid beta protein precursor. J Alzheimer's Dis 2000; 2: 289-301.
38. Slack C, Giannakou ME, Foley A, Goss M, Partridge L. dFOXO-independent effects of reduced insulin-like signaling in Drosophila. Aging Cell 2011; 10: 735-748.

39. Nechipurenko IV, Broihier HT. FoxO limits microtubule stability and is itself negatively regulated by microtubule disruption. J Cell Biol 2012; 196: 345-362.

40. Luo X, Puig O, Hyun J, Bohmann D, Jasper $H$. Foxo and Fos regulate the decision between cell death and survival in response to UV irradiation. EMBO J 2007; 26: 380-390.

41. Lehtinen MK, Yuan Z, Boag PR, Yang Y, Villén J, Becker EB et al. A conserved MST-FOXO signaling pathway mediates oxidative-stress responses and extends life span. Cell 2006; 125: 987-1001.

42. Takaishi H, Konishi H, Matsuzaki H, Ono Y, Shirai Y, Saito N et al. Regulation of nuclear translocation of forkhead transcription factor AFX by protein kinase B. Proc Natl Acad Sci USA 1999; 96: 11836-11841.

43. Fu Z, Tindall DJ. FOXOs, cancer and regulation of apoptosis. Oncogene 2008; 27: 2312-2319.

44. Dansen TB, Smits LM, van Triest MH, de Keizer PL, van Leenen D et al. Redox-sensitive cysteines bridge p300/CBP-mediated acetylation and FoxO4 activity. Nat Chem Biol 2009; 5: 664-672.

45. Shen $\mathrm{C}$, Chen Y, Liu H, Zhang K, Zhang T, Lin A et al. Hydrogen peroxide promotes Abeta production through JNK-dependent activation of gamma-secretase. J Biol Chem 2008; 283: $17721-17730$.

46. Biswas SC, Shi Y, Vonsattel JP, Leung CL, Troy CM, Greene LA. Bim is elevated in Alzheimer's disease neurons and is required for beta-amyloid-induced neuronal apoptosis. J Neurosci 2007; 27: 893-900

47. Torroja L, Packard M, Gorczyca M, White K, Budnik V. The Drosophila beta-amyloid precursor protein homolog promotes synapse differentiation at the neuromuscular junction. J Neurosci 1999; 19: 7793-7803.

48. Ma X, Huang J, Yang L, Yang Y, Li W, Xue L et al. NOPO modulates Egr-induced JNK-independent cell death in Drosophila. Cell Res 2012; 22: 425-431.

49. Milan M, Campuzano S, Garcia-Bellido A. Developmental parameters of cell death in the wing disc of Drosophila. Proc Natl Acad Sci USA 1997; 94: 5691-5696.

50. Ma X, Yang L, Yang Y, Li M, Li W, Xue L. dUev1a modulates TNF-JNK mediated tumor progression and cell death in Drosophila. Dev Biol 2013; 380: 211-221.

51. Xue L, Noll M. Dual role of the Pax gene paired in accessory gland development of Drosophila. Development 2002; 129: 339-346.

52. Yuan Z, Becker EB, Merlo P, Yamada T, DiBacco S, Konishi $Y$ et al. Activation of FOXO1 by Cdk1 in cycling cells and postmitotic neurons. Science 2008; 319: 1665-1668.

53. Zhang YW, Wang R, Liu Q, Zhang $\mathrm{H}$, Liao FF, Xu H. Presenilin/gamma-secretasedependent processing of beta-amyloid precursor protein regulates EGF receptor expression. Proc Natl Acad Sci USA 2007; 104: 10613-10618.

(1) (-) $\odot$ Cell Death and Disease is an open-access journal published by Nature Publishing Group. This work is licensed under a Creative Commons Attribution-NonCommercialNoDerivs 3.0 Unported License. The images or other third party material in this article are included in the article's Creative Commons license, unless indicated otherwise in the credit line; if the material is not included under the Creative Commons license, users will need to obtain permission from the license holder to reproduce the material. To view a copy of this license, visit http://creativecommons.org/licenses/ by-nc-nd/3.0/

Supplementary Information accompanies this paper on Cell Death and Disease website (http://www.nature.com/cddis) 